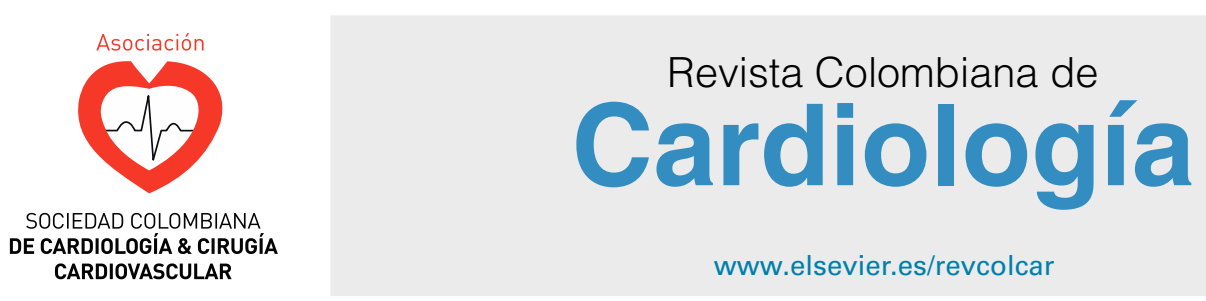

CARDIOLOGÍA DEL ADULTO - ARTÍCULO ORIGINAL

\title{
Masas cardiacas: registro de la experiencia en un centro de alta complejidad
}

\author{
Jairo Gándara Ricardo a ${ }^{\text {, Edison Muñoz Ortiz }}{ }^{\mathrm{b}}$, Edwin Arévalo Guerrero ${ }^{\mathrm{b}}$, \\ Alfonso Mejía Vélez ${ }^{\mathrm{a}, \mathrm{b}}$, José Agamez Gómez ${ }^{\mathrm{a}}$ y Juan $M$. Sénior ${ }^{\mathrm{b}, c, \mathrm{~d}, *}$
}

\author{
a Universidad de Antioquia, Medellín, Colombia \\ ${ }^{\mathrm{b}}$ Hospital Universitario San Vicente Fundación, Medellín, Colombia \\ c Posgrado Cardiología Clínica y Cardiología Intervencionista, Universidad de Antioquia, Medellín, Colombia \\ d Grupo para la Investigación de Enfermedades Cardiovasculares, Universidad de Antioquia, Medellín, Colombia
}

Recibido el 19 de noviembre de 2017; aceptado el 11 de abril de 2018

Disponible en Internet el 17 de julio de 2018

\section{PALABRAS CLAVE \\ Tumores cardiacos; Tumores secundarios; Mixoma}

\begin{abstract}
Resumen
Introducción: Las masas cardiacas son una entidad heterogénea, que incluye lesiones neoplásicas y no neoplásicas y se clasifican en primarias y secundarias o metastásicas. Métodos: Estudio observacional analítico de tipo cohorte retrospectivo.

Resultados: Se incluyeron un total de 73 individuos de los cuales se analizaron los datos de 66. Las masas cardiacas se diagnosticaron con mayor frecuencia en mujeres (53\%). El síntoma más frecuente fue disnea (35\%), seguido por dolor torácico (28\%); otras manifestaciones comunes fueron fiebre $(22 \%)$, pérdida de peso $(22 \%)$ y focalización neurológica $(22 \%)$. 44 pacientes fueron llevados a intervención quirúrgica o toma de biopsia de lesiones primarias encontrando como principal diagnóstico mixoma en 18 de ellos (27\%); el resto de neoplasias primarias cardiacas fueron raras, fibroelastoma 2 casos (3\%), al igual que rabdomioma y rabdomiosarcoma con solo un caso; el compromiso secundario fue frecuente con 18 casos $(27 \%)$ representados principalmente por linfomas con 5 casos (8\%), tumores primarios renales con 4 casos $(6 \%)$ y cáncer de pulmón con 3 casos (5\%). Dentro del seguimiento, el $77 \%$ estaban vivos en los siguientes seis meses del diagnóstico; la principal causa de muerte fue la progresión de la enfermedad oncológica (8 pacientes), seguida por ataque cerebrovascular (3 pacientes) y choque postoperatorio (2 pacientes).
\end{abstract}

\footnotetext{
* Autor para correspondencia. Grupo para el Estudio de las Enfermedades Cardiovasculares, Sección Cardiología, Departamento de Medicina Interna, Universidad de Antioquia, Medellín, Colombia.

Correo electrónico: mmbt@une.net.co (J.M. Sénior).
} 
Conclusiones: Los pacientes con masas cardíacas con frecuencia tienen síntomas inespecíficos incluidos en tres ejes principales: insuficiencia cardíaca, síntomas generales y embolia sistémica. La frecuencia de mixoma auricular y las neoplasias secundarias fue similar.

(C) 2018 Sociedad Colombiana de Cardiología y Cirugía Cardiovascular. Publicado por Elsevier España, S.L.U. Este es un artículo Open Access bajo la licencia CC BY-NC-ND (http:// creativecommons.org/licenses/by-nc-nd/4.0/).

\section{KEYWORDS}

Cardiac tumours; Secondary tumours; Myxoma

\section{Introducción}

Las masas cardiacas son una entidad heterogénea que incluye lesiones neoplásicas y no neoplásicas y se clasifican en primarias y secundarias o metastásicas; los tumores primarios a su vez, en benignos y malignos. La incidencia general, según diferentes series de autopsias, varía entre $0,0017 \%$ a $0,28 \%$, es decir, 17 a 2.800 tumores primarios en un millón de autopsias ${ }^{1}$. Los tumores cardíacos primarios benignos corresponden al 75 a $90 \%$ de los casos y los malignos a un 10 a 25\%. De los tumores primarios benignos, el 25 al 50\% corresponden a mixomas, y de los malignos el $75 \%$ son sarcomas, de los cuales el $20 \%$ corresponden a angiosarcomas. A pesar de ser una entidad rara, sus consecuencias pueden ser catastróficas, mediadas por presentaciones clínicas graves como síncope, falla cardiaca, embolia pulmonar y sistémica e incluso la muerte ${ }^{2}$.

La información acerca de la prevalencia, las características clínicas, los métodos diagnósticos, las opciones terapéuticas y la supervivencia de los pacientes es escasa en nuestro medio; el Instituto Nacional de Cancerología y la Clínica Shaio reportaron una serie de 28 casos de tumores cardíacos primarios, de los cuales el 85\% (21 casos) fueron mixomas, $5 \%$ sarcomas ( 1 caso), $5 \%$ leiomiosarcoma ( 1 caso) y $9,5 \%$ no determinados ( 2 casos), recogidos en el período comprendido entre 1935 y 1994³ la Clínica Cardiovascular Santa María reporta la experiencia de 15 años con 33 casos con diagnóstico histológico; en el 75\% se demostró mixoma ${ }^{4}$. La Fundación Cardioinfantil reportó 34 tumores cardiacos, 24 con confirmación histológica, de los cuales $54 \%$ fueron mixomas $^{5}$.

La escasa información sobre las características de los tumores cardiacos en nuestro medio, incluyendo los secundarios o metastásicos, motivan la realización de esfuerzos que permitan comprender las características clínicas y ecocardiográficas así como las alternativas terapéuticas aplicadas a los pacientes que se diagnostican con masas cardiacas y su evolución. El objetivo de este estudio es describir las características clínicas, ecocardiográficas y terapéuticas, así como el pronóstico de un grupo de pacientes con masas cardiacas diagnosticadas en un centro de alta complejidad de atención en la ciudad de Medellín Colombia.

\section{Métodos}

\section{Tipo de estudio}

Estudio observacional de tipo cohorte retrospectivo. 


\section{Población}

Cohorte retrospectiva de pacientes mayores de un año de edad atendidos en diferentes servicios (Pediatría, Medicina interna y Cardiología) de una institución de alta complejidad de la ciudad de Medellín desde enero de 2000 hasta diciembre de 2015, en quienes de forma incidental o por sospecha clínica se estableció el diagnóstico de masa cardiaca.

\section{Tamaño de la muestra}

Se analizó una muestra no probabilística por conveniencia consistente en la cohorte descrita.

\section{Recolección de datos}

Para el estudio se incluyeron todos los mayores de un año que tuvieran diagnóstico de masa cardiaca en la revisión de los registros físicos de los servicios de ecocardiografía y resonancia magnética, además en los registros electrónicos hospitalarios por medio del sistema de codificación diagnóstica CIE 10 para los siguientes códigos diagnósticos: tumor benigno del corazón (E800), tumor benigno del corazón (D151), tumor maligno del corazón (C380), fibroelastosis endocárdica (1424). Se excluyeron aquellos en quienes no se logró determinar el estatus vital a los seis meses u otra información relevante como las características ecocardiográficas o clínicas. El estatus vital y las otras características de interés se establecieron a través de los registros en la historia clínica o por medio telefónico si era necesario. La información recolectada por los investigadores fue ingresada a una base de datos electrónica (Microsoft Excel) y fue analizada con el paquete estadístico STATA 13. El estudio se considera sin riesgo de acuerdo con la normatividad vigente y se ajusta a las normas de Helsinki.

\section{Análisis estadístico}

Se describieron las características clínicas, paraclínicas y sociodemográficas de los pacientes con masa cardiaca por medio de métodos estadísticos acorde con la naturaleza de las variables. Para las variables categóricas se obtuvieron distribuciones de frecuencias y porcentajes y para las cuantitativas se calcularon las medidas de tendencia central y dispersión de acuerdo con la forma de la distribución. El desenlace principal evaluado en el seguimiento a seis meses fue mortalidad. No se realizó imputación de datos.

\section{Resultados}

La revisión de los registros físicos y electrónicos entre enero de 2000 y diciembre de 2015 arrojó un total de 73 individuos con diagnóstico primario de masa cardiaca; siete de ellos (10\%) se excluyeron por ausencia de datos fundamentales de la ecocardiografía o del seguimiento clínico. Finalmente se analizaron los datos de 66 pacientes.

\section{Características basales}

El promedio de edad fue de $46 \pm 22$ años (rango entre 1$86)$; solo 5 pacientes tenían menos de 18 años. Hubo predominio del diagnóstico de masa intracardiaca en el sexo femenino con un 53\%. Dentro de los síntomas al momento del diagnóstico el más frecuente fue la disnea en el 35\% de los casos, la cual no fue grave en la mayoría de ellos, con una clasificación de NYHA I en el 63\%, NYHA II en el $12 \%$, NYHA III en $8 \%$ y disnea en reposo en el $14 \%$ de los casos; el dolor de pecho fue el segundo síntoma más frecuente, manifiesto en el $28 \%$ de los casos; otros síntomas de presentación importante fueron fiebre y pérdida de peso, ambos en el $22 \%$ de los casos, astenia y adinamia presentes en el $20 \%$ de los pacientes y focalización neurológica en $22 \%$ de los casos. Otra manifestación destacada fue el síncope en el $14 \%$ de los pacientes. Las comorbilidades más importantes estaban representadas por la fibrilación atrial que ocurrió en $11 \%$ de los casos; seis pacientes se encontraban en diálisis $(9 \%)$, de ellos 5 tenían como diagnóstico principal trombo intracavitario y solo un paciente en diálisis tenía mixoma (fig. 1).

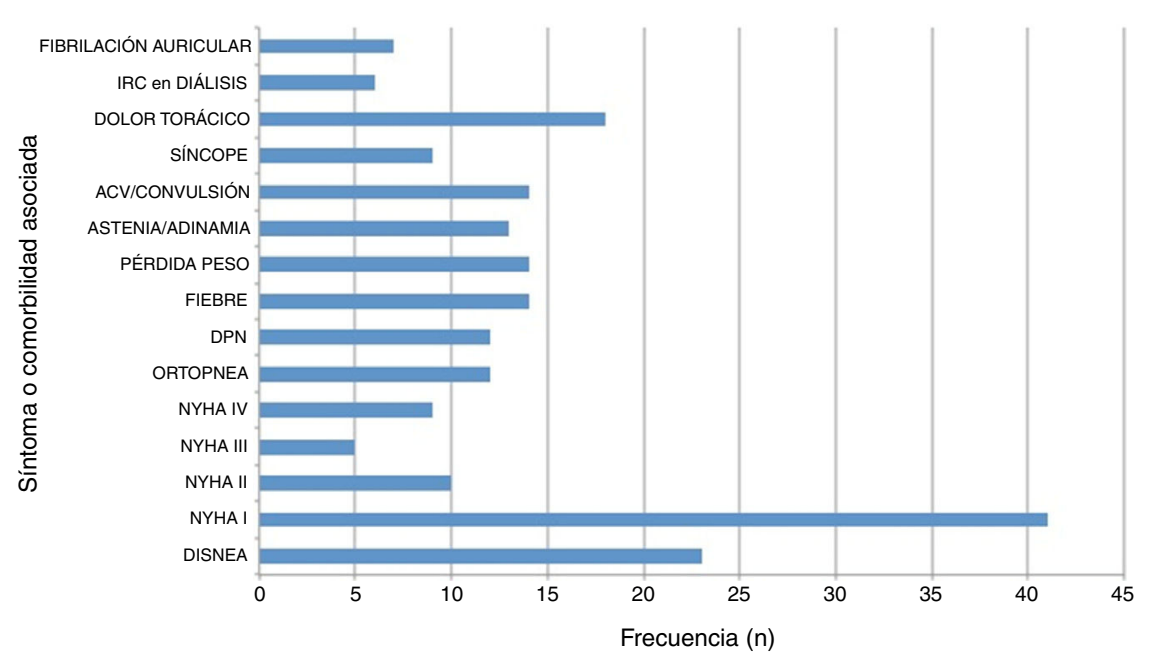

Figura 1 Síntomas y comorbilidades en pacientes con masas intracardiacas *IRC: insuficiencia renal crónica; ACV: ataque cerebrovascular; NYHA: clasificación de la New York Heart Association por síntomas que incluyen disnea, fatiga o angina; DPN: disnea paroxística nocturna. 


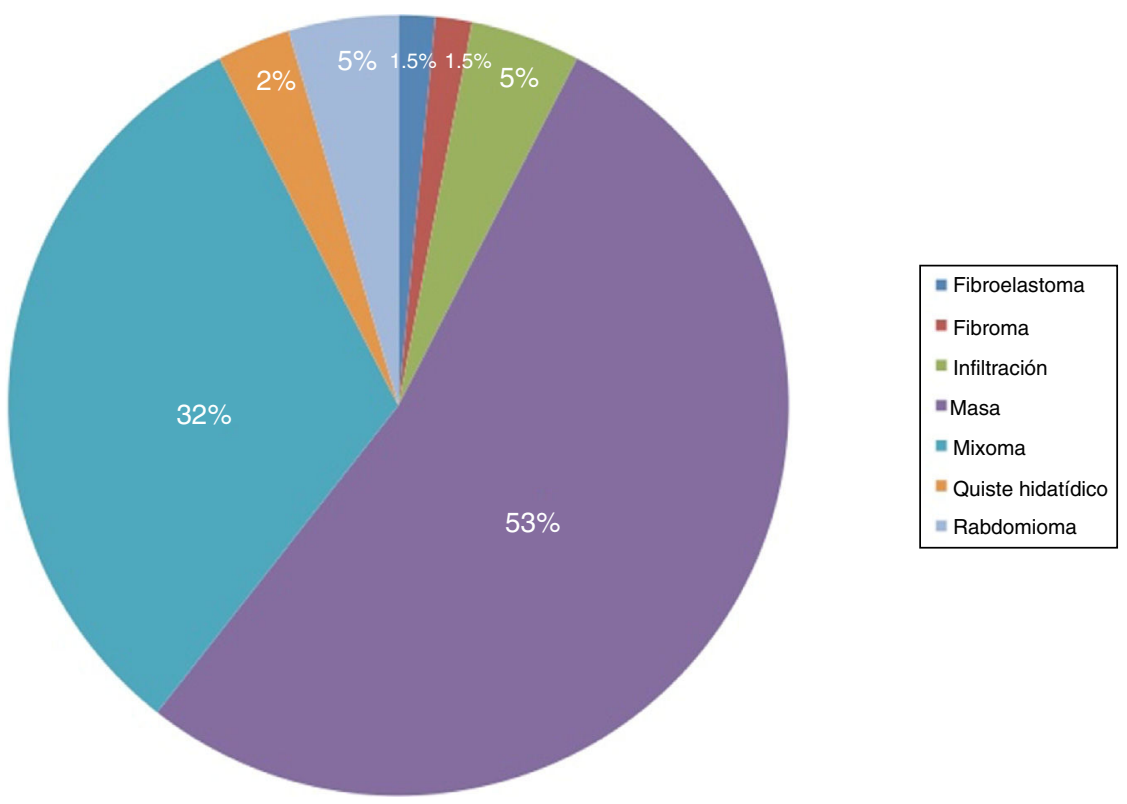

Figura 2 Diagnóstico ecocardiográfico de masa intracardiaca *Diagnóstico en la evaluación ecocardiográfica.

\section{Características ecocardiográficas}

La ecocardiografía se realizó en el $100 \%$ de los pacientes incluidos. La sospecha imagenológica inicial fue masa intracardiaca genérica en 35 pacientes (53\%) seguida por mixoma en 21 pacientes (32\%), infiltración tumoral en 3 pacientes (5\%) al igual que rabdomioma; en dos pacientes se sospechó quiste hidatídico (3\%) y en uno fibroelastoma. La localización más frecuente de la masa al momento de su diagnóstico imagenológico fue la aurícula izquierda en 24 pacientes (37\%), seguido por la aurícula derecha en 19 casos con 29\%; la localización biauricular se dio en 4 pacientes (6\%) (fig. 2).

La localización ventricular fue infrecuente con solo 6 casos en el ventrículo izquierdo (9\%) y 4 casos en el ventrículo derecho (6\%). Entre los otros datos ecocardiográficos importantes está el promedio de fracción de eyección del ventrículo izquierdo que fue de $59 \pm 14 \%$ (20-87\%), en tanto que el promedio de presión sistólica de la arteria pulmonar fue de $50 \mathrm{~mm} \mathrm{Hg}$ (rango entre 20-102 mm Hg) (fig. 3). Las lesiones valvulares graves asociadas fueron la estenosis mitral en 4 casos (6\%), tres de ellas con diagnóstico final de trombo intracavitario y solo una con mixoma auricular, seguida por insuficiencia tricúspide en tres pacientes (4\%) e insuficiencia mitral en 2 pacientes (3\%).

\section{Tratamiento}

El tratamiento más ofrecido fue la cirugía en 33 pacientes $(50 \%)$, en uno de ellos combinada con quimioterapia. El tratamiento médico se continuó sin intervenciones en 26 pacientes (39\%), que incluyó el tratamiento de la enfermedad de base y el alivio de los síntomas; la quimioterapia sola se ofreció en 7 pacientes que representan el $11 \%$.

\section{Diagnóstico definitivo}

La confirmación histológica de la impresión diagnóstica de masa cardiaca por imágenes se obtuvo en 48 pacientes (73\%), ya fuera por cirugía cardiovascular o toma de muestra de la lesión primaria encontrando como principal diagnóstico mixoma en 18 de ellos (27\%); el resto de neoplasias cardiacas primarias fueron raras: el fibroelastoma con 2 casos (3\%) al igual que el rabdomioma y el rabdomiosarcoma con solo un caso. El compromiso secundario fue frecuente con 18 casos (27\%) representados principalmente por linfomas en 5 casos (8\%); tumores primarios renales en 4 casos (6\%) y cáncer de pulmón en 3 casos (5\%). En 12 pacientes el diagnóstico definitivo de la masa cardiaca fue trombo intracavitario y en 4 casos la imagen diagnóstica inicial no se logró corroborar en estudios posteriores, por lo cual se consideró imagen fantasma.

\section{Seguimiento}

Dentro del seguimiento a los treinta días, 55 pacientes (83\%) estaban vivos. En los siguientes seis meses del diagnóstico, el $77 \%$ de los pacientes continuaban en este estatus, lo que representa una mortalidad del $24 \%(16 / 66)$. La principal causa de muerte fue la progresión de la enfermedad oncológica en $12 \%$ de los pacientes, seguido por ataque cerebrovascular en 3 pacientes $(4.5 \%)$ y choque postoperatorio en 2 casos (3\%); un paciente murió por falla cardiaca aguda y otro por sepsis en el periodo postoperatorio; solo 3 pacientes con mixoma (17\%) murieron, 2 casos como consecuencia de ataque cerebrovascular y uno en el contexto de choque postoperatorio. Se reportó recidiva tumoral en 6 pacientes (9\%), no recidivas en 27 casos (41\%) y no se informaron los datos en 33 pacientes (50\%). 


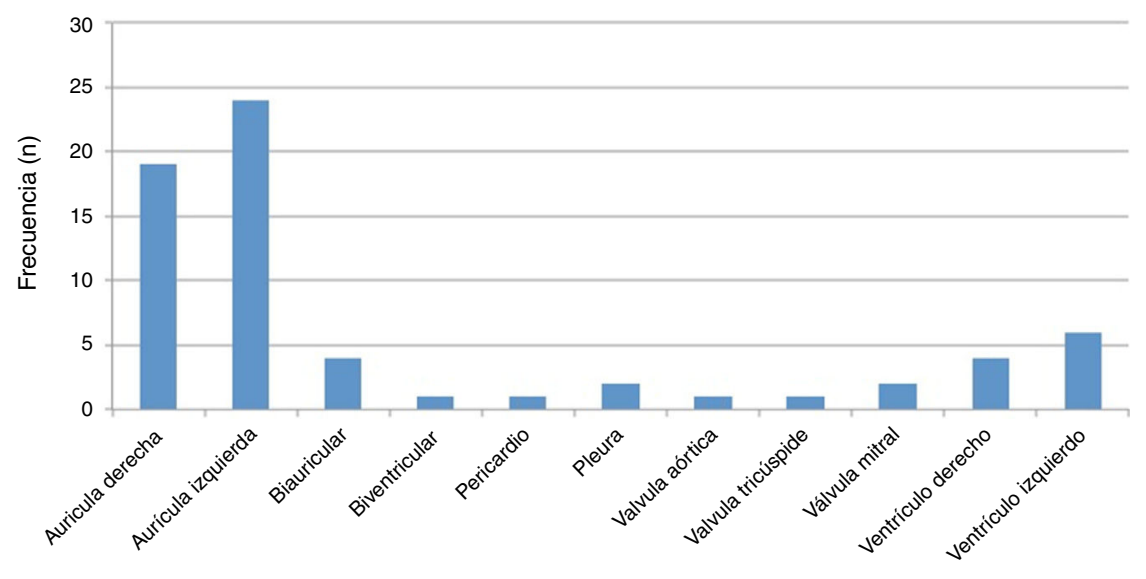

Localización

Figura 3 Localización de las masas intracardiacas y compromiso extracardiaco

*Se describe la localización más frecuente y el compromiso de estructuras extracardíacas por el tumor primario o por contigüidad.

En el análisis de regresión logística se encontró relación significativamente estadística con el desenlace definido de mortalidad con las siguientes variables: edad (OR 0,81 IC 95\% 0,69-0,95, $p=0,013$ ) y presión sistólica de la arteria pulmonar (OR 0,88 IC95\% 0,78-0,99, p=0,046).

\section{Discusión}

Las masas cardiacas son sin duda una entidad diversa tanto en sus características clínicas, como en los hallazgos ecocardiográficos y el comportamiento pronóstico. Una de estas características es la edad de presentación; en este registro la edad promedio fue de $49 \pm 22$ años, hallazgo acorde con los registros locales e internacionales ${ }^{3-6}$. El rango de edad fue amplio, entre 1 a 86 años, lo que manifiesta la pluralidad en el momento de la presentación de estas lesiones. Dentro de los pacientes pediátricos el diagnóstico más frecuente fue rabdomioma en 4 de los 7 casos; si bien son pocos casos, la tendencia es similar a la de otros registros, hecho que posiciona a este tumor como el más frecuente en la edad pediátrica ${ }^{7,8}$. En contraste al rabdomioma, el mixoma se presentó de manera más uniforme a lo largo de todas las edades, con un rango de presentación entre 18 a 84 años. La distribución por sexo es similar, $47 \%$ para hombres y $53 \%$ para mujeres, dato que contrasta con lo encontrado en el registro local previo, en el cual la mayoría se presentaba en el sexo masculino ${ }^{4}$.

Los tumores cardiacos suelen tener un curso indolente, con pocos síntomas y en muchas ocasiones son descubiertos de forma incidental; sin embargo se ha reportado estatus completamente asintomático en menos del $20 \%{ }^{9}$ y los pacientes suelen manifestar algún síntoma relacionado con quejas cardiovasculares, síndrome constitucional o eventos embólicos ${ }^{10,11}$; en esta cohorte, 55 pacientes $(83 \%)$ tuvieron al menos un síntoma de los reportados en la figura 1, y de forma general se pueden agrupar en síntomas de insuficiencia cardiaca (disnea, ortopnea o disnea paroxística nocturna), síntomas generales (fiebre, escalofrío y pérdida de peso) y fenómenos embólicos (ataque cerebrovascular principalmente) con frecuencia y distribución similar a la reportada por otros autores. Piazza et al. informan la disnea como el síntoma más frecuente en $38 \%$ de 21 pacientes diagnosticados con masas cardiacas; esta fue también el síntoma más común en la cohorte que aquí se reporta con porcentaje similar ( $35 \%$ de los casos) ${ }^{12}$. Conviene destacar que los síntomas usualmente se relacionan con su localización en el corazón más que con su histología específica; por ejemplo, los mixomas y los fibroelastomas se relacionan con eventos embólicos. En nuestra cohorte se documentaron 14 ataques cerebrovasculares de los cuales 6 (42\%) estuvieron relacionados con mixomas, correspondiente a un porcentaje del $33 \%$ para este tumor, cifra superior a la informada por otros autores; Wang et al. reportan focalización neurológica en 15,5\% de 207 pacientes con mixomas diagnosticados y tratados quirúrgicamente entre los años 1988 y 2014 en un centro cardiovascular de alta complejidad ${ }^{13}$, porcentaje similar al informado por Pinede et al. quienes muestran que la focalización neurológica ocupó el 20,5\% de las manifestaciones clínicas de 112 casos de mixomas en el periodo comprendido entre 1959-1998 ${ }^{14}$.

La localización más frecuente para las masas fue la aurícula izquierda, probablemente relacionada con mayor número de mixomas cuya implantación habitual es esta cavidad en un $90 \%$ de los $\operatorname{casos}^{11,15}$; en esta cohorte, 14 mixomas de los 18 se alojaron en la aurícula izquierda (78\% de los casos), hallazgo similar a los reportados previamente y representaron el $58 \%$ de todas las masas localizadas en esta cavidad. Otra lesión localizada predominantemente en la aurícula izquierda fue el trombo, con 6 casos $(29 \%), 3$ de ellos relacionados con estenosis mitral, con lo cual se destaca el potencial trombogénico de esta valvulopatía ${ }^{16}$. La localización ventricular fue menos frecuente, con 10 masas que representaron el $15 \%$ de las lesiones; sin embargo fue más del doble de lo reportado por Uribe et al. ${ }^{4}$, de ahí que en nuestro reporte se resalte el compromiso del ventrículo izquierdo en 6 casos, cavidad que no presentó lesiones en el estudio mencionado. Las lesiones localizadas en los ventrículos no presentaron asociación específica puesto que todas las masas alojadas en estas cavidades eran de causas diferentes. 
El compromiso secundario fue frecuente en 18 casos (27\%); no obstante es menor a la proporción reportada por otras series en las que se describe el compromiso metastásico como la principal causa de lesiones cardiacas con 1,5 a $21 \%$ y compromiso primario menos común, en 0,001 a $0,28 \%$ de los análisis de autopsias ${ }^{17}$; de forma inusual, las metástasis cardiacas son la primera manifestación de una enfermedad oncológica primaria de otro sistema y la mayoría de veces es inherente el compromiso avanzado de la enfermedad ${ }^{18}$. En esta cohorte son los linfomas, con 5 casos ( $27 \%$ de las lesiones secundarias), la lesión secundaria más frecuente, en contraposición a otros informes que señalan su presentación en menos del $10 \%$ para esta neoplasia y sitúan las neoplasias de riñón ${ }^{19}$ y los sarcomas ${ }^{17}$ como las lesiones secundarias más comunes, distribución que puede explicarse por la alta complejidad del hospital y centro de referencia para diagnóstico y tratamiento del cáncer hematológico. Los tumores primarios renales completaron 3 casos (5\%), todos localizados en la aurícula derecha en relación probable con sus mecanismos de diseminación hematógena y colonización del corazón por la vena cava inferior y finalmente 3 casos de cáncer de pulmón (5\%) como terceros en frecuencia de presentación, hallazgos que distan de lo reportado en la otra serie local, en la cual solo encontraron 2 lesiones de origen secundario, explicado probablemente por ser centro de remisión cardiovascular exclusiva, y que las patologías tumorales malignas se derivan usualmente a otras instituciones para el diagnóstico y tratamiento interdisciplinario.

El tratamiento usual de las masas intracardiacas implica cirugía, especialmente cuando en forma clínica e imagenológica se presume el diagnóstico de tumor primario; la mayoría de ocasiones incluye la programación rápida de la resección en busca de mitigar el riesgo de embolización sistémica y complicaciones cardiovasculares que incluyen incluso la muerte súbita ${ }^{20,21}$. Los resultados de la resección quirúrgica generalmente son muy buenos, y la mayoría de las series informan mortalidad operatoria inferior al $5 \%{ }^{22,23}$. El tratamiento quirúrgico se ofreció en menos de la mitad de todos los casos, con un alto porcentaje de manejo médico (33\%), señalando que la intervención quirúrgica en las masas cardiacas es un verdadero reto que exige gran versatilidad por parte del cirujano, especialmente en tumores secundarios con posibilidades de curación o remisión. Dentro de las intervenciones están las resecciones simples, la resección compleja y el autotrasplante cardiaco; en nuestra cohorte todas las intervenciones realizadas fueron resecciones simples. El tratamiento de control de síntomas en 26 pacientes (39\%) y la quimioterapia sola en 7 pacientes (11\%), constituyeron en conjunto el $50 \%$ del tratamiento definitivo; esto está relacionado con el número importante de masas explicadas por compromiso secundario que representa estadios avanzados de la enfermedad primaria y trombos intracavitarios; ambos escenarios con escasa frecuencia, tienen indicación del uso de estrategias intervencionistas para tratamiento definitivo.

La supervivencia reportada para los tumores cardiacos es variable, y depende básicamente de la etiología, en cuyo caso es muy favorable para tumores benignos primarios. Pinede et al. analizan los datos de 112 pacientes con mixomas auriculares llevados a cirugía, en 99 de los cuales recuperaron la información del estatus vital al año; solo 4 murieron durante este periodo con una supervivencia del $96 \%{ }^{14}$. De forma similar, Hoffmeier et al. informan los resultados de 78 pacientes con mixomas intervenidos mediante cirugía, con una supervivencia al año superior al 95, 88 y $85 \%$ a los 5 y 10 años, respectivamente ${ }^{24}$. Por el contrario, los pacientes con tumores malignos primarios exhiben una mortalidad del $40 \%$ al seguimiento a los 12 meses, la mayoría relacionada con la extensión metastásica de la enfermedad ${ }^{24}$. Otros estudios ilustran el mal pronóstico de los tumores malignos primarios; por ejemplo Ramlawi et al. reportan una serie de 95 pacientes con tumores cardíacos primarios malignos, todos los cuales recibieron tratamiento quirúrgico con $60 \%$ de quimioterapia adyuvante preoperatoria; al final del análisis solo 2 pacientes estaban vivos al seguimiento a más de cinco años ${ }^{25}$; Oliveira, en un estudio de 40 años de recolección de datos e información de más de 500 pacientes con tumores cardíacos malignos primarios, reportó tasas de supervivencia global en 1, 3 y 5 años del 46, 22 y 17\%, con peores desenlaces para los sarcomas con tasas de supervivencia que fueron del 47, 16 y $11 \%$, respectivamente ${ }^{26}$. En nuestra cohorte la mortalidad a 6 meses fue del 24\%, lo que demuestra la heterogeneidad de la población, que incluyó pacientes con tumores primarios y compromiso secundario de otras neoplasias malignas; sin embargo, no se pudo demostrar asociación entre mortalidad y clasificación del tumor, posiblemente por el pequeño tamaño de muestra y el seguimiento a solo 6 meses, aunque sí se definió asociación con la edad y la presión sistólica de la arteria pulmonar, que están en relación con la distribución del tipo de tumor de acuerdo con el grupo etario y el impacto sobre la reología pulmonar.

\section{Limitaciones}

La limitación más importante es inherente al carácter retrospectivo de la cohorte; pese a ello, dado el seguimiento estrecho que se les realiza por protocolo y a la disponibilidad de historia clínica electrónica, además del seguimiento por consulta ambulatoria y por llamadas telefónicas, la información perdida fue mínima. Se decidió no hacer imputación de datos dada la naturaleza de los mismos.

\section{Conclusiones}

Los pacientes con tumores cardiacos con frecuencia presentan síntomas inespecíficos incluidos en tres ejes principales: falla cardiaca, síntomas generales y embolia sistémica. A diferencia de lo reportado en otras series, la frecuencia del mixoma auricular y el compromiso secundario fue igual para nuestra serie, con un tratamiento definitivo inclinado a la terapia médica y el control de síntomas, probablemente explicado por el compromiso metastásico y el origen no neoplásico de un grupo importante de lesiones. Así mismo, el pronóstico vital es diferente al reportado hasta el momento, quizá por la diversidad de lesiones y la supervivencia final matizada entre lesiones benignas y metastásicas.

\section{Financiación}

Grupo para el Estudio de las Enfermedades Cardiovasculares. 


\section{Conflictos de interés}

Ninguno.

\section{Bibliografía}

1. Reynen K. Frequency of primary tumors of the heart. Am J Cardiol. 1996;77:107.

2. Maleszewski JJ, Anavekar NS, Moynihan TJ, Klarich KW. Pathology, imaging, and treatment of cardiac tumours. Nat Rev Cardiol. 2017;14:536-49, http://dx.doi.org/ 10.1038/nrcardio.2017.47.

3. Ortiz C, Melgarejo I, Arboleda F, Roa C. Tumores primarios del corazón. Sci cardiol Shaio. 1995;15:8-25.

4. Uribe CE, Andrés F, Gómez C. Tumores cardiacos: registro de 15 años de experiencia en la Clínica Cardiovascular Santa María. Rev Colomb Cardiol. 2005;12:45-7.

5. Cubides C, Salazar G, Muñoz A, Pedraza J, Hernandez E, Martinez J, Mugnier J. Tumores cardiacos primarios. Rev Col Cardiol. 2003;10:472-85.

6. Bruckner BA, Reardon MJ. Benign cardiac tumors: a review. Methodist Debakey Cardiovasc J. 2010;6:20-6.

7. Yadava OP. Cardiac tumours in infancy. Indian Heart J. 2012;64:492-6, http://dx.doi.org/10.1016/j.ihj.2012.05.004.

8. Burke A, Virmani R. Pediatric heart tumors. Cardiovasc Pathol. 2008;17:193-8, http://dx.doi.org/10.1016/ j.carpath.2007.08.008.

9. Grebenc ML, Rosado de Christenson ML, Burke AP, Green CE, Galvin JR. Primary cardiac and pericardial neoplasms: radiologic-pathologic correlation. Radiographics. 2000;20: 1073-103.

10. Bjessmo S, Ivert T. Cardiac myxoma: 40 years' experience in 63 patients. Ann Thorac Surg. 1997;63:697-700.

11. Burke A, Jeudy J Jr, Virmani R. Cardiac tumours: An update. Heart. 2008;94:117-23, http://dx.doi.org/10.1136/ hrt.2005.078576.

12. Piazza N, Chugtai T, Toledano K, Sampalis J, Liao C, Morin JF. Primary cardiac tumors: eighteen years of surgical experience on 21 patients. Can J Cardiol. 2004;20:1443-8.

13. Wang $Z$, Chen $\mathrm{S}$, Zhu M, Zhang $\mathrm{W}$, Zhang $\mathrm{H}$, Li $\mathrm{H}$, et al. Risk prediction for emboli and recurrence of primary cardiac myxomas after resection. J Cardiothorac Surg. 2016;11:22, http://dx.doi.org/10.1186/s13019-016-0420-4.

14. Pinede L, Duhaut P, Loire R. Clinical Presentation of Left Atrial Cardiac Myxoma A Series Of 112 Consecutive Cases. Medicine (Baltimore). 2001;80:159-72.
15. Reynen K. Cardiac Myxomas. N Engl J Med. 1995;333:1610-7.

16. Chandrashekhar Y, Westaby S, Narula J. Mitral stenosis. Lancet. 2009;374:1271-83, http://dx.doi.org/10.1016/ S0140-6736(09)60994-6.

17. Neragi-Miandoab S, Kim J, Vlahakes GJ. Malignant Tumours of the Heart: A Review of Tumour Type, Diagnosis and Therapy. Clin Oncol. (R Coll Radiol). 2007;19:748-56, http://dx.doi.org/10. 1016/j.clon.2007.06.009.

18. Sosinska-Mielcarek K, Senkus-Konefka E, Jassem J, Kulczycka J, Jendrzejewski J, Jaskiewicz K. Cardiac Involvement at Presentation of Non-Small-Cell Lung Cancer. J Clin Oncol. 2008;26:1008-10, http://dx.doi.org/10. $1200 /$ JCO.2007.14.9328.

19. Yusuf SW, Bathina JD, Qureshi S, Kaynak HE, Banchs J, Trent JC, et al. Cardiac tumors in a tertiary care cancer hospital: clinical features, echocardiographic findings, treatment and outcomes. Heart Int. 2012;7:e4, http://dx.doi.org/10.4081/hi.2012.e4.

20. Selkane C, Amahzoune B, Chavanis N, Raisky O, Robin J, Ninet J, Obadia JF. Changing Management of Cardiac Myxoma Based on a Series of 40 Cases with Long-Term Follow-Up. Ann Thorac Surg. 2003;76:1935-8.

21. Keeling IM, Oberwalder $P$, Anelli-Monti M, Schuchlenz $H$, Demel U, Tilz GP, et al. Cardiac myxomas: 24 Years of experience in 49 patients. Eur J Cardiothorac Surg. 2002;22: 971-7.

22. Centofanti P, Di Rosa E, Deorsola L, Dato GM, Patanè F, La Torre M, et al. Primary cardiac tumors: early and late results of surgical treatment in 91 patients. Ann Thorac Surg. 1999;68:1236-41.

23. Wang $Y$, Wang $X$, Xiao $Y$. Surgical treatment of primary cardiac valve tumor: early and late results in eight patients. J Cardiothorac Surg. 2016;11:31, http://dx.doi.org/10. 1186/s13019-016-0406-2.

24. Hoffmeier A, Schmid C, Deiters S, Drees G, Rothenburger $M$, Tjan TDT, et al. Neoplastic heart disease - The Muenster experience with 108 patients. Thorac Cardiovasc Surg. 2005;53:1-8, http://dx.doi.org/10.1055/s-2004-830389.

25. Ramlawi B, Leja MJ, Abu Saleh WK, Al Jabbari O, Benjamin R, Ravi V, et al. Surgical Treatment of Primary Cardiac Sarcomas: Review of a Single-Institution Experience. Ann Thorac Surg. 2016;101:698-702, http://dx.doi.org/10.1016/ j.athoracsur.2015.07.087.

26. Oliveira G, Al-Kindi S, Hoimes C, Park S. Characteristics and survival of malignant cardiac tumors. A 40 year analysis of $>500$ patients. Circulation. 2015;132:2395-402, http://dx.doi.org/10.1161/CIRCULATIONAHA.115.016418. 